\title{
Effect of Adding Dexmedetomidine as Adjuvant to Different Regional Anesthetic Techniques after Inguinal Herniorrhaphy
}

\author{
Ezzat Mahmoud Ali El-Saudi, Waheed Mohamed Ali, Esraa Mohamed Mohamed Abd-elsalam*
}

Department of Anesthesiology and Intensive Care, Faculty of Medicine - Al-Azhar University

*Corresponding author: Esraa Mohamed Mohamed Abd-elsalam, Mobile: (+20)1096399632,

E-Mail: esr.m.ab@gmail.com

\section{ABSTRACT}

Background: Inguinal herniorrhaphy is one of the most commonly performed surgeries, often performed on a fast-track basis. However, inguinal herniorrhaphy is frequently associated with persistent postoperative discomfort and pain, which can lead to patient distress, delayed discharge, and subsequent complication.

Objective: Aim of this work is to compare TAP block technique versus local infiltration with or without dexmedetomidine regarding analgesic effect and endogenous stress response.

Patients and Methods: This randomized prospective study included 120 patients of both genders scheduled for non-complicated inguinal hernioplasty. Their age ranged between 18 and 60 years, with ASA physical status I and II, and body mass index $\leq 35 \mathrm{~kg} / \mathrm{m}^{2}$. The study was approved by the medical ethics committee of Al-Azhar University Hospital in Assiut and a written informed consent is obtained from all patients.

Results: The major finding in this study was that the pain scores were statistically significantly lower when we added dexmedetomidine than when we did not add it and in the surgical site infiltration groups than TAP block groups at postoperative $2^{\text {nd }}, 6^{\text {th }}$, and $12^{\text {th }}$ hours. $1^{\text {st }}$ request for analgesia in this study among all groups was similar. Frequency of analgesic doses was statistically significant among all groups, and group $\mathrm{T}$ used the most frequent doses. In this study, local wound infiltration and TAP block with dexmedetomidine showed decrease number of analgesic doses and attenuated the stress response indicators (norepinephrine and glucose levels) without side effects.

Conclusion: Dexmedetomidine added to bupivacaine in both local infiltration and TAP block had better visual analogue scale, decreased number of analgesic doses and attenuated postoperative stress response indicators. Norepinephrine is the most accurate stress response indicator while blood glucose is accurate and the cheapest one. Keywords: Dexmedetomidine, Regional anesthetic techniques, Inguinal herniorrhaphy

\section{INTRODUCTION}

Post-herniorrhaphy pain is conventionally treated with non-steroidal anti-inflammatory drugs (NSAIDS) or opioids. However, these drugs can induce certain side effects, such as gastrointestinal adverse events, postoperative bleeding, vomiting, respiratory depression and sedation ${ }^{(1)}$.

They eventually lead to delayed discharge from hospital, thus attenuating the advantage of fast-track basis surgery for which early postoperative pain relief is imperative. Therefore, a continuous search is underway to reduce the use of post-operative analgesics, such as ilioinguinal nerve blockade (2), caudal blockade ${ }^{(3)}$, systemic administration or infiltration of local anesthetics (LA) directly into the wound ${ }^{(4)}$, and transversus abdominis plane block (TAP) ${ }^{(5)}$.

Local and/or regional analgesia techniques are critical components of an optimal multimodal analgesia techniques, as they have been shown to improve pain relief, as well as reduce opioid requirement. Surgical site local anesthetic infiltration has been shown to provide excellent analgesia and is recommended, when appropriate. Transversus abdominis plane blocks have been increasingly used in patient undergoing lower abdominal surgical procedures because of improved pain relief and reduced opioid requirement ${ }^{(6)}$.

Dexmedetomidine is a newly developed selective alpha 2-adrenoceptor (AR) agonist used as a sedative or adjuvant anesthetic. The results of a recent study demonstrated that synergistic interactions exist between dexmedetomidine and LA.

Systemic administration of dexmedetomidine enhanced spinal and epidural anesthesia ${ }^{(7)}$, intravenous regional anesthesia ${ }^{(8)}$, and its addition to LA prolonged the duration of blockade and enhanced the analgesic and anesthetic property in a caudal and sciatic nerve block ${ }^{(9)}$.

Various animal studies have been conducted using intrathecal dexmedetomidine at a dose range of 2.5 to $100 \mu \mathrm{g}$ without any neurological complications (10). Antinociceptive effect of dexmedetomidine, a highly selective alpha 2 adrenergic agonist was evaluated in animal studies. Dexmedetomidine was used to enhance the analgesic property of local anesthetics like lidocaine, bupivacaine and ropivacaine. In vivo and in vitro studies indicated that these local anesthetics had significant neurotoxicity (11). Dexmedetomidine showed protective or growth promoting properties in tissues, including nerve cells from cortex and has a neuroprotective effect similar to methylprednisolone in spinal cord injury when used intrathecally ${ }^{(\mathbf{1 2})}$.

\section{AIM OF THE WORK \\ To compare local infiltration versus transversus abdominal plane block (TAP) with or}


without adding dexmedetomidine as regard the analgesic effect and endogenous stress response.

\section{PATIENTS AND METHODS \\ Ethical approval:}

This randomized prospective clinical trial was approved by the medical ethics committee of AlAzhar University Hospital in Assiut. Written informed consent is obtained from all patients.

This study included 120 patients of both genders scheduled for non-complicated inguinal hernioplasty, age ranged between 18 and 60 years, with ASA physical status I and II, and body mass index $\leq$ $35 \mathrm{~kg} / \mathrm{m}^{2}$.

\section{Exclusion criteria:}

- Patients with sensitivity to local anesthetics,

- Uncontrolled systemic disease: hepatic, renal, coronary artery disease,

- Endocrine diseases and diabetes mellitus,

- Patients on corticosteroid therapy,

- bleeding tendency,

- emergent or complicated hernia,

- Inability to obtain written informed consent (patient refusal or psychological diseases).

\section{Group Allocation:}

Patients who fulfilled the inclusion criteria were allocated randomly using computer generated random table into four equal groups each of 30 patients.

- Group LD: underwent local infiltration at the end of surgery by bupivacaine with dexmedetomidine,

- Group L: underwent local infiltration at the end of surgery by bupivacaine without dexmedetomidine,

- Group TD: underwent TAP at the end of surgery by bupivacaine with dexmedetomidine

- Group T: underwent TAP at the end of surgery by bupivacaine without dexmedetomidine.

Preoperatively, patients were taught in how to evaluate their own pain intensity using the visual analogue scale (VAS), scored from 0-10 (where $0=$ no pain and $10=$ worst pain imaginable). Preoperative sedation was avoided and plasma levels of epinephrine and norepinephrine, serum level of cortisol and blood level of glucose were measured.

\section{Anesthetic technique (for all groups):}

The anesthetic technique was standardized in all groups. Anesthesia was induced with fentanyl $1 \mu \mathrm{g} / \mathrm{kg}, 2 \mathrm{mg} / \mathrm{kg}$ of propofol IV and $0.1 \mathrm{mg} / \mathrm{kg}$ of cisatracurium IV. The trachea was intubated and ventilation was controlled at a tidal volume of $6-7 \mathrm{ml} / \mathrm{kg}$ and at a respiratory rate of 12 breaths/min. Anesthesia was maintained using $1.2 \%$ isoflurane in $3 \mathrm{l} / \mathrm{min} \mathrm{O} 2$ and $0.25 \mathrm{mg} / \mathrm{kg}$ of cisatracurium every $30 \mathrm{~min}$. . During surgery, patients received an IV infusion of Ringer's solution at a rate of $3-6 \mathrm{ml} / \mathrm{kg} / \mathrm{h}$. No additional analgesics were injected during the surgery. Heart rate, pulse oximetry and systolic, diastolic and mean blood pressure were monitored all the time of operation.

Group LD and L (local wound infiltration groups).

After repair of muscle and before closure of wound, all layers of the surgical incision were infiltrated with a 22-gauge, 40-mm needle in a controlled and systemic manner under direct visualization in fanlike fashion on each side of incision. In group LD, $20 \mathrm{ml}$ of $\mathbf{0 . 2 5 \%}$ isobaric bupivacaine added by $0.5 \mu \mathrm{g} / \mathrm{kg}$ of dexmedetomidine was used. In group L, $20 \mathbf{~ m l}$ of $\mathbf{0 . 2 5 \%}$ isobaric bupivacaine was used.

\section{Group TD and T (TAP block groups).}

\section{Ultrasound guided TAP block technique:}

The TAP block was performed with the use of ultrasound under complete aseptic technique. Images were obtained using a Sonosite M-Turbow ultrasound machine (Sonosite Inc., Bothell, WA, USA) with a10$5 \mathrm{MHz} 38 \mathrm{~mm}$ broadband linear array probe. The ultrasound probe was placed transversely to the abdomen (horizontal plane) in the midaxillary line midway between the costal margin and the iliac crest. Three muscle layers are clearly seen in the image. The needle (Sono Plex Stim cannula (PAJUNK) $22 \mathrm{G} \times 80 \mathrm{~mm}$, GERMANY) was inserted in a sagittal plane approximately $3-4 \mathrm{~cm}$ medial to the ultrasound probe. For optimal imaging of the needle it should be held parallel to the long the needle tip was directed into the plane below the internal oblique and above the transversus abdominis muscle. For confirmation of proper needle placement, a small volume of normal saline $(2 \mathrm{ml})$ was seen to open the plane between the two muscles and was followed by insertion of the full dose of local anesthetic. If the $2 \mathrm{ml}$ dose appears to be within muscles rather than between them, needle adjustment was required. In group TD, $20 \mathrm{ml}$ of $\mathbf{0 . 2 5 \%}$ isobaric bupivacaine added by $0.5 \mu \mathrm{g} / \mathrm{kg}$ of dexmedetomidine was injected. In group T, $20 \mathrm{ml}$ of $\mathbf{0 . 2 5 \%}$ isobaric bupivacaine was injected. The local anesthetic injectate appeared hypoechoic (black compared to the muscle layers) on ultrasound image.

Then reversal of muscle relaxant by neostigmine $(0.05 \mathrm{mg} / \mathrm{kg})$ and atropine $(0.01 \mathrm{mg} / \mathrm{kg})$ and extubation was performed when the patient met the following criteria: hemodynamic stability, adequate muscle strength, full consciousness, and adequate ventilation (respiratory rate: 10 to $30 \mathrm{breath} / \mathrm{min}, \mathrm{SpO} 2$ : $94 \%$ or more). All patients were transmitted to recovery room until become fully recovered.

Postoperatively, all patients were admitted to post anesthesia care unit (PACU). The patient's heart rate and non-invasive arterial blood pressure were monitored for the first 24 hour postoperatively and the values were recorded at $2^{\text {nd }}, 4^{\text {th }}, 6^{\text {th }}, 12^{\text {th }}$, and 24 th hours. The severity of pain, nausea, vomiting, and respiratory depression were assessed postoperatively. Plasma levels of epinephrine and norepinephrine, serum level of cortisol and blood level of glucose were measured as indicator of endogenous stress response at $2^{\text {nd }}$ and $6^{\text {th }}$ 
hours postoperatively. The severity of pain was assessed using a $10-\mathrm{cm}$ visual analogue scale $(0=$ no pain and $10=$ worst imaginable pain). Rescue analgesia was given for visual analogue scale (VAS) $\geq 3$ by IV infusion of $15 \mathrm{mg} / \mathrm{kg}$ paracetamol. Time to the first rescue analgesia and the frequency of the given drug in the first 24 hours, and also nausea and vomiting were recorded. Rescue antiemetic (10 mg metocloperamide) was given for any patient who complained of vomiting. Signs of side effects was recorded: hypotension (defined as mean arterial blood pressure $\leq 60 \mathrm{mmHg}$ ) and this was managed by IV fluids infusion and intravenous boluses of ephedrine $0.1 \mathrm{mg} / \mathrm{kg}$, bradycardia (defined as heart rate $\leq 55$ beats/min) was managed by IV atropine $0.01 \mathrm{mg} / \mathrm{kg}$ and respiratory depression (arterial O2 saturation $\leq 94 \%$ ) and this will be managed by supplementary $\mathrm{O} 2$ delivered by face mask. For assessment of sedation we used Ramsay scale. In the $1^{\text {st }} 3$ degrees of the scale we considered patient not sedated while in the last 3 degrees of the scale we considered patient sedated.

\section{Data collection:}

- Preoperative patient's data including age, weight and gender was collected.

- $\quad$ Systolic, diastolic and mean arterial blood pressure and heart rate were recorded before surgery,

- Plasma level of epinephrine and norepinephrine, serum level of cortisone and blood level of glucose were measured night before surgery.

- $\quad$ VAS scores were collected at $2^{\text {nd }}, 6^{\text {th }}, 12^{\text {th }}$ and $24^{\text {th }}$ hours postoperatively.

- Time to the first rescue analgesia and its frequency were recorded.

- Systolic, diastolic and mean arterial blood pressure and heart rate were recorded at $2^{\text {nd }}, 4^{\text {th }}, 6^{\text {th }}, 12^{\text {th }}$, and $24^{\text {th }}$ hours postoperatively.

- Venous sample was withdrawn at $2^{\text {nd }}$ and $6^{\text {th }}$ hours postoperatively for detection of plasma level of epinephrine and norepinephrine, serum level of cortisone and blood level of glucose.
- $\quad$ Side effects as hypotension, bradycardia, respiratory depression, sedation and vomiting were recorded.

\section{Statistical analysis}

Data were verified, coded by the researcher and analysed using the Statistical Package for Social Sciences (IBM-SPSS ver. 21).

Descriptive statistics: Means, standard errors, medians and percentages were calculated. Test of significances: Chi square test was used to compare the difference in distribution of frequencies among different groups. For continuous variables with more than two categories; ANOVA test was calculated to test the mean differences of the data that follow normal distribution, post-hoc test was calculated to calculate pairwise differences using Bonferroni corrections. Repeated Measure ANOVA test was used to compare the differences in mean values over the study time. A p-value equal to or less than 0.05 was considered statistically significant.

\section{RESULTS}

During 14 months from January 2017 to March 2018, this randomized controlled study included one hundred and twenty patients scheduled for elective inguinal hernioplasty surgery. The study aimed at comparing the effect of adding dexmedetomedine to TAP block and local infiltration versus not adding dexmedetomedine to TAP block and local infiltration on VAS, hemodynamics, and the inflammatory stress response.

For this purpose patients were randomly allocated into four groups: local infiltration with dexmedetomedine (group LD) $(n=30)$, local infiltration without dexmedetomedine (group L) $(n=30)$, TAP block with dexmedetomedine (group TD) $(n=30)$, TAP block without dexmedetomedine (group T) $(n=30)$.

\section{General patient characteristics (table 1):}

Patients' general characteristics were summarized in table (1). All groups were comparable to each other as regard age, gender, and weight.

Table (1): Comparison between the different groups regarding to general characteristics.

\begin{tabular}{|c|c|c|c|c|c|}
\hline & $\begin{array}{c}\text { G LD } \\
(n=30)\end{array}$ & $\begin{array}{c}\mathbf{G ~ L} \\
(\mathbf{n}=\mathbf{3 0})\end{array}$ & $\begin{array}{c}\text { G TD } \\
(n=30)\end{array}$ & $\begin{array}{c}\mathbf{G ~ T} \\
(\mathrm{n}=30)\end{array}$ & P-value \\
\hline $\begin{array}{ll} & \text { Age/years } \\
\bullet & \text { Mean } \pm \text { SD }\end{array}$ & $37.87 \pm 2.4$ & $36.60 \pm 2.2$ & $35.37 \pm 2.1$ & $36.47 \pm 2.2$ & $=0.836^{*}$ \\
\hline Gender n (\%) & & & & & \multirow{3}{*}{$=0.232 * * *$} \\
\hline - Female & $7(23.3 \%)$ & $2(6.7 \%)$ & $4(13.3 \%)$ & $3(10.0 \%)$ & \\
\hline - Male & $23(76.7 \%)$ & $28(93.3 \%)$ & $26(86.7 \%)$ & $27(90.0 \%)$ & \\
\hline $\begin{array}{r}\text { Weight/kg } \\
\text { - Mean } \pm \text { SD }\end{array}$ & $71.30 \pm 13.1$ & $70.03 \pm 10.7$ & $69.10 \pm 12.1$ & $72.53 \pm 8.8$ & $=0.675^{*}$ \\
\hline
\end{tabular}

\footnotetext{
*ANOVA test was used to compare the means among groups

**Post-hoc analysis with Bonferroni corrections. ***Chi-square analysis was used to compare the proportions between groups
} 


\section{Postoperative VAS (Table 2)}

There was significant difference among all groups from $2^{\text {nd }}$ hour postoperatively until $12^{\text {th }}$ hour postoperatively. P-value for VAS at $2^{\text {nd }}$ hour was 0.014 while it was 0.045 for VAS at $6^{\text {th }}$ hour and it was $<0.001$ for VAS at $12^{\text {th }}$ hour. Patients in group LD showed the lowest VAS value at $2^{\text {nd }}, 6^{\text {th }}$ and $12^{\text {th }}$ hours postoperatively. At $24^{\text {th }}$ hour, there was no significant difference among all groups with $\mathrm{P}$-value $=0.359$.

Table (2): Comparison of the VAS among the different groups

\begin{tabular}{|c|c|c|c|c|c|}
\hline & $\begin{array}{c}\text { G LD } \\
(n=30)\end{array}$ & $\begin{array}{c}\mathbf{G} \mathbf{L} \\
(\mathbf{n}=\mathbf{3 0})\end{array}$ & $\begin{array}{c}\text { G TD } \\
(\mathbf{n}=30)\end{array}$ & $\begin{array}{c}\text { G T T } \\
(\mathbf{n}=\mathbf{3 0})\end{array}$ & P-value \\
\hline \multicolumn{2}{|c|}{ VAS after 2 hours } & & & & \multirow{2}{*}{$=0.014 *$} \\
\hline Mean \pm SD & $0.47 \pm 0.1$ & $0.83 \pm 0.1$ & $0.70 \pm 0.1$ & $1.17 \pm 0.3$ & \\
\hline P-value** & $\begin{array}{c}\text { LD vs } \mathrm{L}=\mathbf{0 . 0 1 1} \\
\mathrm{LD} \text { vs } \mathrm{TD}=\mathbf{0 . 0 3 4}\end{array}$ & $\begin{array}{c}\mathrm{L} \text { vs TD }=0.186 \\
\mathbf{L} \text { vs } \mathbf{T}=\mathbf{0 . 0 0 6}\end{array}$ & TD vs $T=0.002$ & LD vs $T<0.001$ & \\
\hline \multicolumn{2}{|c|}{ VAS after 6 hours } & & & & \multirow{2}{*}{$=0.045 *$} \\
\hline Mean \pm SD & $1.53 \pm 0.2$ & $1.93 \pm 0.3$ & $1.60 \pm 0.2$ & $2.37 \pm 0.3$ & \\
\hline P-value** & $\begin{array}{c}\text { LD vs } \mathbf{L}=\mathbf{0 . 0 3 3} \\
L D \text { vs } T D=0.117\end{array}$ & $\begin{array}{c}\mathrm{L} \text { vs } \mathrm{TD}=0.060 \\
\mathrm{~L} \text { vs } \mathrm{T}=0.069\end{array}$ & TD vs $T=0.016$ & LD vs $T=0.011$ & \\
\hline \multicolumn{2}{|c|}{ VAS after 12 hours } & & & & \multirow{2}{*}{$<0.001 *$} \\
\hline Mean \pm SD & $2.90 \pm 0.2$ & $3.47 \pm 0.3$ & $3.07 \pm 0.5$ & $3.90 \pm 0.5$ & \\
\hline P-value** & $\begin{array}{c}L D \text { vs } L=0.011 \\
L D \text { vs } T D=0.021 \\
\end{array}$ & $\begin{array}{l}\mathrm{L} \text { vs } \mathrm{TD}=0.137 \\
\mathrm{~L} \text { vs } \mathrm{T}=0.087\end{array}$ & TD vs $T=0.042$ & LD vs $T=0.004$ & \\
\hline \multicolumn{2}{|c|}{ VAS after 24 hours } & & & & \multirow{2}{*}{$=0.359 *$} \\
\hline Mean \pm SD & $2.20 \pm 0.4$ & $2.67 \pm 0.6$ & $2.43 \pm 0.5$ & $2.90 \pm 0.7$ & \\
\hline P-value** & $\begin{array}{c}\mathbf{L D} \text { vs } \mathbf{L}=\mathbf{0 . 0 4 2} \\
L D \text { vs } T D=0.075\end{array}$ & $\begin{array}{c}\mathrm{L} \text { vs } \mathrm{TD}=0.214 \\
\mathrm{~L} \text { vs } \mathrm{T}=0.158\end{array}$ & TD vs $\mathrm{T}=0.398$ & LD vs $T=0.013$ & \\
\hline
\end{tabular}

*ANOVA test was used to compare the means among groups

***ost-hoc analysis with Bonferroni corrections

$* * *$ Repeated Measure ANOVA test was used to compare the means over study time

\section{$1^{\text {st }}$ analgesic request Time and Number of Analgesic Doses (Table 3)} $>0.05$

The time for $1^{\text {st }}$ analgesic request had no statistically significant differences among all groups with P-value

Although there was no statistically significant difference between group LD and group L or group TD, there was statistically significant difference between group LD and group $\mathrm{T}$.

There was significant difference among four groups in total analgesic dose with $\mathrm{P}$ - value $<0.05$.

Table (3): Comparison of $1^{\text {st }}$ Dose timing and number of analgesic doses among the studied groups

\begin{tabular}{|c|c|c|c|c|c|}
\hline & $\begin{array}{c}\text { G LD } \\
(\mathbf{n}=\mathbf{3 0})\end{array}$ & $\begin{array}{c}\mathbf{G} \mathbf{L} \\
(\mathbf{n}=\mathbf{3 0})\end{array}$ & $\begin{array}{c}\text { G TD } \\
(\mathbf{n}=\mathbf{3 0})\end{array}$ & $\begin{array}{c}\mathbf{G} \mathbf{T} \\
(\mathbf{n}=\mathbf{3 0})\end{array}$ & P-value \\
\hline $1^{\text {st }}$ Analgesia Time & & & & & \multirow{2}{*}{$\begin{array}{c}= \\
0.079 *\end{array}$} \\
\hline Mean \pm SD & $7.60 \pm 0.7$ & $7.30 \pm 0.3$ & $6.80 \pm 0.5$ & $5.87 \pm 0.3$ & \\
\hline \multirow[t]{2}{*}{ P-value** } & $\mathrm{LD}$ vs $\mathrm{L}=0.671$ & $\mathrm{~L}$ vs $\mathrm{TD}=0.479$ & TD vs $\mathrm{T}=0.188$ & LD vs $T=0.015$ & \\
\hline & $\mathrm{LD}$ vs $\mathrm{TD}=0.259$ & L vs T $=0.044$ & & & \\
\hline No. of Doses & & & & & \multirow{2}{*}{$\begin{array}{c}= \\
0.004 *\end{array}$} \\
\hline Mean \pm SD & $1.77 \pm 0.2$ & $2.07 \pm 0.1$ & $1.80 \pm 0.1$ & $2.33 \pm 0.1$ & \\
\hline \multirow[t]{2}{*}{ P-value** } & LD vs $\mathrm{L}=0.087$ & $\mathrm{~L}$ vs $\mathrm{TD}=0.128$ & TD vs $\mathrm{T}=\mathbf{0 . 0 0 3}$ & LD vs $\mathrm{T}=\mathbf{0 . 0 0 1}$ & \\
\hline & LD vs $\mathrm{TD}=0.848$ & $\mathrm{~L}$ vs $\mathrm{T}=0.128$ & & & \\
\hline
\end{tabular}

*ANOVA test was used to compare the means among groups

**Post-hoc analysis with Bonferroni corrections 
Laboratory data:

A. Norepinephrine level (Table 4)

Baseline values in all groups were comparable to each other.

There was significant difference at norepinephrine level at preoperative, $2^{\text {nd }}$ and $6^{\text {th }}$ hours among all groups with $\mathrm{P}$ - value $<0.05$.

Table (4): Comparison of nor-epinephrine level among the groups

\begin{tabular}{|c|c|c|c|c|c|}
\hline & $\begin{array}{c}\text { G LD } \\
(n=30)\end{array}$ & $\begin{array}{c}\text { G L } \\
(n=30)\end{array}$ & $\begin{array}{c}\text { G TD } \\
(n=30)\end{array}$ & $\begin{array}{c}\text { G T } \\
(n=30)\end{array}$ & -value* \\
\hline \multicolumn{2}{|c|}{ NE Level at Baseline } & & & & \multirow{2}{*}{$=\mathbf{0 . 0 3 7}$} \\
\hline Mean \pm SD & $103.50 \pm 20.6$ & $271.20 \pm 48.3$ & $130.30 \pm 27.6$ & $174.30 \pm 8.2$ & \\
\hline \multirow[t]{2}{*}{ P-value ${ }^{* * *}$} & LD vs $\mathbf{L}=\mathbf{0 . 0 0 7}$ & L vs TD $=\mathbf{0 . 0 2 2}$ & TD vs $\mathrm{T}=0.459$ & LD vs $\mathrm{T}=0.236$ & \\
\hline & $\mathrm{LD}$ vs TD $=0.651$ & $\mathrm{~L}$ vs T $=0.108$ & & & \\
\hline \multicolumn{2}{|c|}{ NE Level after 2 hours } & & & & \multirow{2}{*}{$=\mathbf{0 . 0 0 7}$} \\
\hline Mean \pm SD & $93.50 \pm 13.8$ & $231.80 \pm 32.4$ & $123.20 \pm 24.3$ & $187.60 \pm 12.7$ & \\
\hline \multirow[t]{2}{*}{ P-value $* *$} & LD vs $L=0.002$ & L vs TD $=0.011$ & $\mathrm{TD}$ vs $\mathrm{T}=0.119$ & LD vs $\mathrm{T}=\mathbf{0 . 0 2 5}$ & \\
\hline & LD vs TD $=0.466$ & $\mathrm{~L}$ vs $\mathrm{T}=0.280$ & & & \\
\hline \multicolumn{2}{|c|}{ NE Level after 6 hours } & & & & \multirow{2}{*}{$=\mathbf{0 . 0 0 1}$} \\
\hline Mean \pm SD & $90.50 \pm 3.6$ & $244.30 \pm 34.4$ & $119.90 \pm 4.1$ & $178.80 \pm 8.2$ & \\
\hline \multirow[t]{2}{*}{ P-value*** } & LD vs $\mathrm{L}<0.001$ & L vs $\mathrm{TD}=\mathbf{0 . 0 0 2}$ & $\mathrm{TD}$ vs $\mathrm{T}=0.121$ & LD vs $\mathrm{T}=\mathbf{0 . 0 2 3}$ & \\
\hline & $\mathrm{LD}$ vs $\mathrm{TD}=0.433$ & $\mathrm{~L}$ vs $\mathrm{T}=0.085$ & & & \\
\hline P-value ${ }^{* * * *}$ & $=0.323$ & $=0.555$ & $=0.486$ & $=0.807$ & \\
\hline
\end{tabular}

*ANOVA test was used to compare the means among groups

**Post-hoc analysis with Bonferroni corrections

***Repeated Measure ANOVA test was used to compare the means over study time

\section{B. Serum epinephrine level (Table 5)}

There were no significant differences in serum epinephrine level among all groups preoperatively or postoperatively with $\mathrm{P}$-value $>0.05$.

Table (5): Comparison of epinephrine level among the studied groups

\begin{tabular}{|c|c|c|c|c|c|}
\hline & $\begin{array}{c}\text { G LD } \\
(n=30)\end{array}$ & $\begin{array}{c}\text { G L } \\
(n=30)\end{array}$ & $\begin{array}{c}\text { G TD } \\
(n=30)\end{array}$ & $\begin{array}{c}\text { G T } \\
(n=30)\end{array}$ & P-value* \\
\hline \multicolumn{2}{|c|}{ EP Level at Baseline } & & & & \multirow{2}{*}{$=0.910$} \\
\hline - Mean \pm SD & $53.20 \pm 10.3$ & $64.20 \pm 9.1$ & $59.20 \pm 11.3$ & $59.90 \pm 13.6$ & \\
\hline \multirow[t]{2}{*}{ - P-value** } & $\mathrm{LD}$ vs $\mathrm{L}=0.492$ & $\mathrm{~L}$ vs $\mathrm{TD}=0.754$ & $\mathrm{TD}$ vs $\mathrm{T}=0.836$ & $\mathrm{LD}$ vs $\mathrm{T}=0.866$ & \\
\hline & LD vs TD $=0.707$ & $\mathrm{~L}$ vs $\mathrm{T}=0.603$ & & & \\
\hline \multicolumn{2}{|c|}{ EP Level after 2 hours } & & & & \multirow{2}{*}{$=0.511$} \\
\hline - Mean \pm SD & $48.50 \pm 8.1$ & $68.00 \pm 10.4$ & $58.60 \pm 8.3$ & $63.90 \pm 9.7$ & \\
\hline \multirow[t]{2}{*}{ - P-value ${ }^{* *}$} & $\mathrm{LD}$ vs $\mathrm{L}=0.156$ & $\mathrm{~L}$ vs $\mathrm{TD}=0.490$ & $\mathrm{TD}$ vs $\mathrm{T}=0.696$ & $\mathrm{LD}$ vs $\mathrm{T}=0.260$ & \\
\hline & LD vs TD $=0.458$ & $\mathrm{~L}$ vs $\mathrm{T}=0.763$ & & & \\
\hline \multicolumn{2}{|c|}{ EP Level after 6 hours } & & & & \multirow{2}{*}{$=0.152$} \\
\hline - Mean \pm SD & $49.20 \pm 8.6$ & $98.30 \pm 17.5$ & $57.90 \pm 9.1$ & $67.80 \pm 8.2$ & \\
\hline \multirow[t]{2}{*}{ - P-value $* *$} & LD vs $\mathbf{L}=\mathbf{0 . 0 3 3}$ & $\mathrm{L}$ vs TD $=0.067$ & $\mathrm{TD}$ vs $\mathrm{T}=0.657$ & $\mathrm{LD}$ vs $\mathrm{T}=0.406$ & \\
\hline & $\mathrm{L}$ vs TD $=0.696$ & $\mathrm{~L}$ vs T $=0.176$ & & & \\
\hline P-value $* * *$ & $=0.494$ & $=0.353$ & $=0.860$ & $=0.356$ & \\
\hline
\end{tabular}

*ANOVA test was used to compare the means among groups

**Post-hoc analysis with Bonferroni corrections

***Repeated Measure ANOVA test was used to compare the means over study time 


\section{Serum cortisol level (table 6)}

- There was no significant difference in preoperative level among all groups with P-value $>0.05$.

- There was no significant difference in postoperative level at 2 and 6 hours among all groups with P-value $>0.05$.

- There was no significant difference among preoperative and postoperative level among all groups with P-value $>0.05$.

Table (6): Comparison of serum cortisol level among the different groups

\begin{tabular}{|c|c|c|c|c|c|}
\hline & G LD $(n=30)$ & G L $(n=30)$ & G TD $(n=30)$ & G T $(n=30)$ & P-value* \\
\hline \multicolumn{2}{|c|}{ Cortisol Level at Baseline } & & & & \multirow{2}{*}{$=0.110$} \\
\hline - Mean \pm SD & $278.30 \pm 52.9$ & $274.90 \pm 9.8$ & $429.50 \pm 53.9$ & $201.40 \pm 48.7$ & \\
\hline \multirow[t]{2}{*}{ - P-value** } & LD vs $L=0.893$ & $\mathrm{~L}$ vs $\mathrm{TD}=0.101$ & TD vs $T=0.018$ & $\mathrm{LD}$ vs $\mathrm{T}=0.356$ & \\
\hline & LD vs $T D=0.130$ & $\mathrm{~L}$ vs $\mathrm{T}=0.492$ & & & \\
\hline \multicolumn{2}{|c|}{ Cortisol Level after 2 hours } & & & & \multirow{2}{*}{$=0.993$} \\
\hline - Mean \pm SD & $228.50 \pm 57.7$ & $232.60 \pm 49.3$ & $248.70 \pm 41.1$ & $244.60 \pm 69.2$ & \\
\hline \multirow[t]{2}{*}{ - P-value** } & $\mathrm{LD}$ vs $\mathrm{L}=0.958$ & $\mathrm{~L}$ vs $\mathrm{TD}=0.838$ & TD vs $\mathrm{T}=0.958$ & $\mathrm{LD}$ vs $\mathrm{T}=0.838$ & \\
\hline & LD vs $L=0.789$ & $\mathrm{TD}$ vs $\mathrm{T}=0.879$ & & & \\
\hline \multicolumn{2}{|c|}{ Cortisol Level after 6 hours } & & & & \multirow{2}{*}{$=0.981$} \\
\hline - Mean \pm SD & $253.00 \pm 62.7$ & $235.40 \pm 51.5$ & $232.60 \pm 45.8$ & $263.50 \pm 57.9$ & \\
\hline \multirow[t]{2}{*}{ - P-value ${ }^{* *}$} & $\mathrm{LD}$ vs $\mathrm{L}=0.837$ & $\mathrm{~L}$ vs $\mathrm{TD}=0.974$ & TD vs $\mathrm{T}=0.718$ & $\mathrm{LD}$ vs $\mathrm{T}=0.912$ & \\
\hline & LD vs $T D=0.812$ & $\mathrm{~L}$ vs $\mathrm{T}=0.748$ & & & \\
\hline P-value ${ }^{* * *}$ & $=0.347$ & $=0.393$ & $=0.007$ & $=0.159$ & \\
\hline
\end{tabular}

*ANOVA test was used to compare the means among groups

**Post-hoc analysis with Bonferroni corrections

***Repeated Measure ANOVA test was used to compare the means over study time

\section{Blood glucose level (Table 7)}

- There was no significant difference in preoperative level among all groups with $\mathrm{P}$ - value $>0.05$.

- At $2^{\text {nd }}$ hour and $6^{\text {th }}$ hours postoperative, there was significant difference among all groups with $P$-value $<0.05$.

- At $2^{\text {nd }}$ hour there was significant difference between group LD and groups L and T.

- At $6^{\text {th }}$ hour there was significant difference between group LD and groups L and T.

Table (7): Comparison of blood glucose level among the studied groups

\begin{tabular}{|c|c|c|c|c|c|}
\hline & G LD $(n=30)$ & G L $(n=30)$ & G TD $(n=30)$ & G T $(n=30)$ & P-value* \\
\hline \multicolumn{5}{|c|}{ Glucose Level at Baseline } & \multirow{2}{*}{$=0.890$} \\
\hline - Mean \pm SD & $131.60 \pm 11.8$ & $131.20 \pm 5.7$ & $123.50 \pm 12.1$ & $133.70 \pm 8.1$ & \\
\hline \multirow[t]{2}{*}{ - P-value ${ }^{* *}$} & LD vs $\mathrm{L}=0.977$ & $\mathrm{~L}$ vs $\mathrm{TD}=0.582$ & TD vs $\mathrm{T}=0.466$ & $L D$ vs $T=0.880$ & \\
\hline & LD vs TD $=0.562$ & $\mathrm{~L}$ vs $\mathrm{T}=0.858$ & & & \\
\hline \multicolumn{3}{|c|}{ lucose Level after 2 hours } & & & \multirow{2}{*}{$=\mathbf{0 . 0 0 8}$} \\
\hline - Mean \pm SD & $109.90 \pm 7.3$ & $133.70 \pm 5.3$ & $123.20 \pm 5.5$ & $136.80 \pm 4.1$ & \\
\hline \multirow[t]{2}{*}{ - P-value** } & LD vs $L=0.005$ & L vs TD $=0.199$ & TD vs $\mathrm{T}=0.099$ & LD vs $\mathrm{T}=\mathbf{0 . 0 0 2}$ & \\
\hline & LD vs TD $=0.106$ & $\mathrm{~L}$ vs $\mathrm{T}=0.702$ & & & \\
\hline \multicolumn{3}{|c|}{ lucose Level after 6 hours } & & & \multirow{2}{*}{$=0.026$} \\
\hline - Mean \pm SD & $117.60 \pm 8.5$ & $148.80 \pm 7.3$ & $135.60 \pm 5.1$ & $138.70 \pm 6.6$ & \\
\hline \multirow[t]{2}{*}{ - P-value ** } & LD vs $L=0.003$ & L vs TD $=0.190$ & TD vs $\mathrm{T}=0.756$ & LD vs $\mathbf{T}=\mathbf{0 . 0 4 0}$ & \\
\hline & LD vs TD $=0.077$ & $\mathrm{~L}$ vs $\mathrm{T}=0.314$ & & & \\
\hline P-value $* * * *$ & $=0.248$ & $=0.046$ & $=0.240$ & $=0.702$ & \\
\hline
\end{tabular}

*ANOVA test was used to compare the means among groups, **Post-hoc analysis with Bonferroni corrections

$* * *$ Repeated Measure ANOVA test was used to compare the means over study time

\section{Incidence of complications (table 8):}

Patients of all groups were followed up postoperatively for incidence of complications. There was no significant difference between all groups as regard vomiting, hypotension and bradycardia with P-value > 0.05 . Incidence of vomiting was comparable in both groups. Hypotension is defined as decrease of more than $20 \%$ of baseline value occurred only in 2 cases in group TD. Bradycardia defined as decreased heart rate less than 50 beats $/ \mathrm{min}$. of baseline was recorded in 3 patients in group LD and only 2 patients in group TD. Sedation has significant difference between groups with $\mathrm{P}$-value $<0.05$. There were 4 cases in group LD and 5 cases in group TD. 
Table (8): Distribution of the study group according to incidence of complications

\begin{tabular}{|c|c|c|c|c|c|}
\hline & $\begin{array}{c}\text { G LD } \\
(\mathbf{n}=30)\end{array}$ & $\begin{array}{c}\text { G L } \\
(n=30)\end{array}$ & $\begin{array}{c}\text { G TD } \\
(n=30)\end{array}$ & $\begin{array}{c}\text { G T } \\
(n=30)\end{array}$ & P-value* \\
\hline \multicolumn{2}{|c|}{ Vomiting n (\%) } & & & & \multirow{3}{*}{$=0.150$} \\
\hline - No & $26(86.7 \%)$ & $25(83.3 \%)$ & $27(90.0 \%)$ & $29(96.7 \%)$ & \\
\hline - Yes & $4(13.3 \%)$ & $5(16.7 \%)$ & $3(10.0 \%)$ & $1(3.3 \%)$ & \\
\hline \multicolumn{2}{|c|}{ Sedation n (\%) } & & & & \multirow{3}{*}{$=0.019$} \\
\hline - No & $26(86.7 \%)$ & $30(100 \%)$ & $25(83.3 \%)$ & $30(100 \%)$ & \\
\hline - Yes & $4(13.3 \%)$ & $0(0 \%)$ & $5(16.7 \%)$ & $0(0 \%)$ & \\
\hline \multicolumn{2}{|c|}{ Hypotension n (\%) } & & & & \multirow{3}{*}{$=0.107$} \\
\hline - No & $30(100 \%)$ & $30(100 \%)$ & $28(93.3 \%)$ & $30(100 \%)$ & \\
\hline - Yes & $0(0 \%)$ & $0(0 \%)$ & $2(6.7 \%)$ & $0(0 \%)$ & \\
\hline \multicolumn{2}{|c|}{ Bradycardia n (\%) } & & & & \multirow{3}{*}{$=0.131$} \\
\hline - No & $27(90.0 \%)$ & $30(100 \%)$ & $28(93.3 \%)$ & $30(100 \%)$ & \\
\hline - Yes & $3(10.0 \%)$ & $0(0 \%)$ & $2(6.7 \%)$ & $0(0 \%)$ & \\
\hline
\end{tabular}

*Chi-square test was used to compare the proportions among groups

**Post-hoc analysis with Bonferroni corrections

\section{DISCUSSION}

In this study, 120 patients of both genders scheduled for non-complicated inguinal hernioplasty. They were divided into four groups: group LD; local infiltration with dexmedetomidine, group L; local infiltration without dexmedetomidine, group TD; TAP with dexmedetomidine, group $\mathrm{T}$; TAP without dexmedetomidine.

The major finding in this study was that the pain scores were statistically significantly lower when we added dexmedetomidine than when we did not add it and in the surgical site infiltration groups than TAP block groups' at postoperative $2^{\text {nd }}, 6^{\text {th }}$, and $12^{\text {th }}$ hours.

$1^{\text {st }}$ request for analgesia in this study among all groups was similar. Frequency of analgesic doses was statistically significant among all groups, and group $\mathrm{T}$ used the most frequent doses.

Our observations can be explained by the fact that in spite of the performance of the TAP blocks with real-time ultrasound, the spread of local anesthetic may not be uniformly consistent because of the presence of anatomical variations ${ }^{(13)}$. In addition, nerves located between the inguinal ligament and the costal margin in the anterior axillary line have variable segmental origin from T9-L1, which may influence the efficacy of TAP blocks ${ }^{(14)}$.

Our results are consistent with previous published reports comparing local wound infiltration and TAP block.

Three studies reported that meticulous local infiltration of all layers of anterior abdominal wall result in better analgesia than ultrasound guided TAP block ${ }^{(15)}$.

In one study by Irina et al. $^{\left({ }^{(6)}\right.}$ comparing the analgesic efficacy of surgical site infiltration with liposomal bupivacaine and bilateral TAP block with $0.5 \%$ bupivacaine in open abdominal hysterectomy. The pain scores at rest and with coughing were significantly lower in the surgical site infiltration group at all postoperative time points $(\mathrm{p}<0.0001)$. The opioid requirements between 24 and 48 hours were significantly lower in infiltration group $(\mathrm{p}=0.009)$. The nausea scores, occurrence of vomiting, and need for rescue analgesia were similar.

In another study by Petersen et al. (16) comparing the analgesic of local wound infiltration, TAP and placebo in inguinal hernia repair, VAS pain score during coughing which was the primary objective was higher in TAP block group than local infiltration group and placebo.

On the other hand, in a systematic review performed by Yu et al. ${ }^{(17)}$, they compared Transversus abdominis-plane block versus local anesthetic wound infiltration in lower abdominal surgery. They concluded that TAP block and LAI provide comparable short-term postoperative analgesia, but TAP block has better long-lasting effect ${ }^{(\mathbf{1 7})}$. This difference in his results from our results may due to change in type of operation.

Dexmedetomidine gave better pain scores in local wound infiltration and TAP block groups (group $\mathrm{LD}$, group $\mathrm{TD}$ )

In a meta-analysis by $\mathbf{Y u}$ et al. ${ }^{(17)}$, they concluded that wound infiltration and TAP block with local anesthetic alone provided short-term postoperative analgesia in lower abdominal surgery.

In this study, local wound infiltration and TAP block with dexmedetomidine showed decrease number of analgesic doses and attenuated the stress response indicators (norepinephrine and glucose levels) without side effects.

Our findings regarding the analgesic efficacy of dexmedetomidine were in accordance with Ulgey $\boldsymbol{e t}$ al. ${ }^{(18)}$ where they found that dexmedetomidine reduced rescue analgesic consumption and provided a better 
pain relief when added to local anesthetic solution infiltrated to the surgical wound.

Luan et al. ${ }^{(19)}$ reported that adding $1.0 \mu \mathrm{g} / \mathrm{kg}$ dexmedetomidine to $0.3 \%$ ropivacaine for wound infiltration prompted the analgesic properties of ropivacaine, reduced sufentanil consumption, and had no effect on wound healing.

In addition, Kang ${ }^{(20)}$ found that a combination of dexmedetomidine and ropivacaine infiltration reduced postoperative pain significantly with no adverse effects after inguinal herniorrhaphy.

In another study by Singh and Prasad (21) concluded that wound infiltration of bupivacaine with dexmedetomidine $1.0 \mu \mathrm{g} / \mathrm{kg}$ provides superior pain relief and decrease in total opioid consumption compared to wound infiltration with bupivacaine alone.

Eldegwy and Alfke ${ }^{(22)}$ reported in their study that using dexmedetomidine as an additive to levobupivacaine in ultrasound-guided TAP block for herniorrhaphy provides prolonged duration of postoperative analgesia, and lowered VAS pain scores. Also, local anesthetic infiltration can give accepted postoperative analgesia but with shorter duration than TAP block.

Performing two different block technique or addition of dexmedetomidine was found to insignificantly affect the investigated hemodynamics. We found insignificant differences in the patients' hemodynamics before and after the block at all investigated period.

Serum norepinephrine level in current study was significantly decreased in addition of dexmedetomidine at postoperative $2^{\text {nd }}$ and $6^{\text {th }}$ hours while epinephrine level show minor decrease which represent insignificant difference. Although serum cortisol level shows insignificant differences among groups, blood glucose level showed significant decrease when adding dexmedetomidine.

It is expected for stress response to surgery to be attenuated by sympatholytic effects of central $\alpha 2$ adrenergic receptor activation, leading to reductions in anti-inflammatory effects. Dexmedetomidine activates receptors in the medullary vasomotor center, reducing norepinephrine turnover, reducing its neuronassociated activity, and decreasing central sympathetic outflow through the medullo-spinal noradrenergic pathway, resulting in alterations in sympathetic function (23). In addition, it was found that dexmedetomidine inhibited the hyperglycemic response to surgery significantly more than placebo, and this may reflect attenuation of sympathoadrenal response ${ }^{(24)}$.

Studies investigating both regional and systemic use of dexmedetomidine have confirmed its surgical stress-suppressing effects; Nasr and Abdelhamid ${ }^{(25)}$ reported that caudal dexmedetomidine attenuated stress response to surgical trauma and provided better postoperative analgesia.
Abd El-Moneim et al. (26) proved that dexmedetomidine alleviated stress response in patients undergoing cancer surgeries, but it was associated with higher sedation.

Khalil et al. ${ }^{(27)}$ also in accordance with our results, found that cortisol and prolactin levels fell during the first postoperative hour in children between 18 months and 10 years old, given $1 \mathrm{~mL} / \mathrm{kg} 0.25 \%$ bupivacaine by caudal block.

Local stress-attenuating effects of dexmedetomidine can be referred to its chemical nature, being an imidazole, which may lead to inhibition of cortisol synthesis; this may participate to its stress-attenuating effects when administered by all routes ${ }^{(28)}$.

However, we believe that stress response attenuation in dexmedetomidine group is principally the result of its local analgesic effects, as pain and stress are mutually interactive. The local site of administration of dexmedetomidine is the main reason for the absence of side effects associated with its systemic use.

Mohamed et al. ${ }^{(29)}$ found in their study that Local wound infiltration with ketamine or dexmedetomidine added to bupivacaine decreased the total dose of morphine consumption, delayed first request of rescue analgesia, and attenuated postoperative stress response, especially with ketamine in patients underwent total abdominal hysterectomy.

Performance of either local infiltration or TAP block technique resulted in no complication related to technique. The use of dexmedetomidine as adjuvant to local anesthetic has insignificant effect on hemodynamics, respiration, and nausea and vomiting. Dexmedetomidine caused sedation in nine cases of total sixty cases in our study.

Mandal et al. (30) found no dizziness, drowsiness or other side effects, in local wound infiltration with dexmedetomidine.

Mohta et al. (31) also reported that dexmedetomidine added to bupivacaine for paravertebral block decreased the incidence of postoperative nausea and vomiting (PONV) in patients who underwent major breast cancer surgery.

\section{Conclusion:}

Dexmedetomidine added to bupivacaine in both local infiltration and TAP block had better visual analogue scale, decreased number of analgesic doses and attenuated postoperative stress response indicators.

Local wound infiltration performed under direct visualization, is a simple and quick technique and it is more effective; on the other hand, ultrasoundguided TAP block is operator-dependent, timeconsuming, and less effective; thus, future researches are required to demonstrate the time requirements and cost efficiency of these two methods.

Norepinephrine is the most accurate stress response indicator while blood glucose is accurate and the cheapest one. 


\section{REFERENCE}

1. Maund E, McDaid C, Rice S, Wright K, Jenkins B, Woolacott N (2011): Paracetamol and selective and non-selective nonsteroidal anti-inflammatory drugs for the reduction in morphinerelated side effects after major surgery: a systematic review. Br J Anesth., 106:292-7.

2. Markham SJ, Tomlinson J, Hain WR (1986): Ilioinguinal nerve block in children. A comparison with caudal block for intra and postoperative analgesia. Anesthesia, 41:1098-103.

3. Ivani G, De Negri P, Lonnqvist PA, Eksborg S, Mossetti V, Grossetti R (2003): A comparison of three different concentrations of levobupivacaine for caudal block in children. Anesth Analg., 97:368-71.

4. Kang H, Kim BG (2011): Intravenous lidocaine for effective pain relief after inguinal herniorrhaphy: a prospective, randomized, double-blind, placebo-controlled study. J Int Med Res., 39:435-45.

5. McDonnell JG, O'Donnell B, Farrell T, Gough N, Tuite D, John G (2007): Transversus abdominis plane block: a cadaveric and radiological evaluation. Reg Anesth Pain Med., 32: 399-404.

6. Irina G, Gohn A, Babatonda O, Charine H, David R, Girish PJ (2015): Tranversus abdominis plane block versus surgical site infiltration for pain management after open total abdominal hysterectomy. International Anesthesia Research Society, 121:1383-6.

7. Kaya FN, Yavascaoglu B, Turker G, Yildirim A, Gurbet A, Mogol EB, Ozcan B (2010): Intravenous dexmedetomidine, but not midazolam, prolongs bupivacaine spinal anesthesia. Can J Anaesth., 57:39-45.

8. Memis D, Turan A, Karamanlioglu B, Pamukcu Z, Kurt I (2004): Adding dexmedetomidine to lidocaine for intravenous regional anesthesia. Anesth Analg., 98:835-40.

9. Brummett CM, Padda AK, Amodeo FS, Welch KB, Lydic $R$ (2009): Perineural dexmedetomidine added to ropivacaine causes a dose-dependent increase in the duration of thermal antinociception in sciatic nerve block in rat. Anesthesiology, 111:1111-19.

10. Smith LJ (2013): A comparison of epidural analgesia provided by bupivacaine alone, bupivacaine and morphine or bupivacaine and dexmedetomidine for pelvic orthopedic surgery in dogs. Vet Anesth Analg., 40(5):527-36.

11. Werdehausen R, Fazeli S, Hollmann MW, Bauer I, Stevens MF (2009): Apoptosis induction by different local anesthetics in a neuroblastoma cell line. Br J Anesth., 103(5):711-8.

12. Celik F, Gocmez C, Kamasak K, Tufek A, Guzel A, Tokqoz O (2013): The comparison of neuroprotective effect of intrathecal dexmedetomidine and methylprednisolone in spinal card injury. Int J Surg., 11(5)414-8.

13. Lew VK, Gray AT (2013): An unusual transversus abdominis plane block: anatomic variation in the internal oblique muscle. Anesthesiology, 119:1209-17.

14. Rozen WM, Tran TM, Ashton MW, Barrington MJ, Ivanusic JJ, Taylor GI (2008): Refining the course of the thoracolumbar nerves: a new understanding of the innervation of the anterior abdominal wall. Clin Anast., 21:325-33.

15. Sakamoto B, Harker G, Eppstein AC, Gwirtz K (2016): Efficacy of local anesthetic with dexamethasone on the quality of recovery following total extraperitoneal bilateral inguinal hernia repair: a randomized clinical trial. JAMA Surgery,151(12):1108-14.

16. Petersen PL, Mathiesen O, Stjernholm P, Kristiansen VB, Torup H, Hansen EG, Mitchell AU, Moeller A, Rosenberg J, Dahl JB (2013): The effect of transversus abdominis plane block or local anesthetic infiltration in inguinal hernia repair A randomized clinical trial. Eur J Anesthesiol., 30:415-421.

17. Yu, N, Long X, Lujan-Hernandez JR, Succar J, Xin X, Wang X (2014): Transversus abdominis-plane block versus local anesthetic wound infiltration in lower abdominal surgery: A systematic review and meta-analysis of randomized controlled trials. BMC Anesthesiol., 14, 121.

18. Ulgey A, Gunes I, Bayram A, Bicer C, Mehmet Kurt F, Muderis I, Boyacı A (2015): The analgesic effects of incisional levobupivacaine with dexmedetomidine after total abdominal hysterectomy. Erciyes Med J., 37: 64-68.

19. Luan H, Zhu P, Zhang X, Tian L, Feng J, Wu Y, Yan Y, Zhao Z, Gu X (2017): Effect of dexmedetomidine as an adjuvant to ropivacaine for wound infiltration in patients undergoing open gastrectomy: A prospective randomized controlled trial. https://www.ncbi.nlm. nih.gov/pmc/articles/PMC5617697/

20. Kang $\mathbf{H}$ (2012): The effect of dexmedetomidine added to preemptive ropivacaine infiltration on post-operative pain after inguinal herniorrhaphy a prospective, randomized, double blind, placebo-controlled study. Eur Surg., 44: 274-280.

21. Singh S, Prasad C (2017): Post-operative analgesic effect of dexmedetomidine administration in wound infiltration for abdominal hysterectomy: A randomized control study. Indian $\mathbf{J}$ Anesth., 61:494-8.

22. Eldegwy MH, Alfkey R (2018): Ultrasound-guided TAP Block with or without dexmedetomidine vs. local infiltration of the wound after open herniorrhaphy. A randomized prospective controlled study. J Anesth Clin Res., 9: 800-5. DOI: 10.4172/2155-6148.

23. Callesen T, Kehlet H (1997): Post herniorrhaphy pain. Anesthesiology, 87:1219-30.

24. Mukhtar AM, Obayah EM, Hassona AM (2006): The use of dexmedetomidine in pediatric cardiac surgery. Anesth Analg., 103: $52-56$.

25. Nasr DA, Abdelhamid HM (2013): The efficacy of caudal dexmedetomidine on stress response and postoperative pain in pediatric cardiac surgery. Ann Card Anaesth., 16: 109-114.

26. Abd El-Moneim NM, Samy MA, Ebrahim WA, Boules NS (2015): Effect of morphine versus low and high dose dexmedetomidine on postoperative stress response in patients undergoing cancer surgeries. Med J Cairo Univ., 83: 41-46.

27. Khalil SN, Hanna E, Farag A, Govindaraj R, Vije H, Kee S, Chuang AZ (2005): Pre-surgical caudal block attenuates stress response in children. Middle East J Anesthesiol., 18: 391-400.

28. Maze M, Virtanen R, Daunt D, Banks SJ, Stover EP, Feldman D (1991): Effects of dexmedetomidine, a novel imidazole sedative anesthetic agent, on adrenal steroidogenesis: In vivo and in vitro studies. Anesth Analg., 73: 204-208.

29. Mohamed SA, Sayed DM, EI Sherif FA, Abd El-Rahman AM (2018): Effect of local wound infiltration with ketamine versus dexmedetomidine on postoperative pain and stress after abdominal hysterectomy, a randomized trial. European Journal of Pain, 22(5):951-60.

30. Mandal D, Das A, Chhaule S, Halder PS, Paul J, Roy Basunia S, Chattopadhyay S, Mandal SK (2016): The effect of dexmedetomidine added to preemptive (2\% lignocaine with adrenaline) infiltration on intraoperative hemodynamics and postoperative pain after ambulatory maxillofacial surgeries under general anesthesia. Anesth Essays and Res., 10: 324-331.

31. Mohta M, Kalra B, Sethi AK, Kaur N (2017): Efficacy of dexmedetomidine as an adjuvant in paravertebral block in breast cancer surgery. J Anesth., 30:252-60. 